\title{
U-Turn Alternating Sign Matrices, Symplectic Shifted Tableaux and their Weighted Enumeration
}

\author{
A.M. HAMEL \\ ahamel@wlu.ca \\ Department of Physics and Computer Science, Wilfrid Laurier University, Waterloo, Ontario N2L 3C5, Canada \\ R.C. KING \\ R.C.King@maths.soton.ac.uk \\ School of Mathematics, University of Southampton, Southampton SO17 1BJ, England
}

Received August 25, 2003; Revised August 2, 2004; Accepted September 8, 2004

\begin{abstract}
Alternating sign matrices with a U-turn boundary (UASMs) are a recent generalization of ordinary alternating sign matrices. Here we show that variations of these matrices are in bijective correspondence with certain symplectic shifted tableaux that were recently introduced in the context of a symplectic version of Tokuyama's deformation of Weyl's denominator formula. This bijection yields a formula for the weighted enumeration of UASMs. In this connection use is made of the link between UASMs and certain square ice configuration matrices.
\end{abstract}

Keywords: alternating sign matrices, symplectic tableaux

\section{Introduction}

Alternating sign matrices with a U-turn boundary (UASMs) first appeared in a paper by Tsuchiya [19] but have been given a wider audience by Kuperberg [9] and Propp [14] (who called them half alternating sign matrices). In this paper we introduce a generalization of UASMs, called $\mu$-UASMs, that combine the U-turn notion with the $\mu$-generalization of alternating sign matrices (ASMs) due to Okada [13], where $\mu$ is a partition all of whose parts are distinct. We show that there exists a natural correspondence between $\mu$-UASMs and the symplectic shifted tableaux of shifted shape $\mu$ defined elsewhere [4], and prove that this correspondence is a bijection.

There exists an important connection between ordinary alternating sign matrices (ASMs) and square ice that was used to provide a second proof of the alternating sign matrix conjecture by Kuperberg [8]. The square ice model involves two-dimensional grids populated by frozen water molecules taking up any one of six configurations, see for example the work of Lieb [11], Bressoud [1] and Lascoux [10]. With a suitable choice of boundary conditions this model can be linked to UASMs in a bijective manner [9]. Here we extend this to the case of $\mu$-UASMs. To make this connection explicit it is convenient to introduce square ice configuration matrices. These are then used to provide both $x$ and $t$-weightings of $\mu$-UASMs, that are an exact counterpart to corresponding weightings of symplectic shifted tableaux.

The significance of this is that it allows a connection to be made with Weyl's formula for characters of irreducible representations of the symplectic Lie algebra $s p(2 n)$, or to be more precise with products of certain $t$-deformations of such characters with a $t$-deformation 
of Weyl's denominator formula [4]. These products are completely determined by the weighted enumeration of symplectic shifted tableaux, so that thanks to the bijection between $\mu$-UASMs and symplectic shifted tableaux derived here we are able to provide a general formula for the weighted enumeration of both $\mu$-UASMs and the UASMs themselves. The latter correspond to the special cases $\mu=\delta=(n, n-1, \ldots, 1)$ for some positive integer $n$. The most basic corollary of our result is

$$
\sum_{U A \in \mathcal{U} \mathcal{A}} 2^{\operatorname{neg}(U A)}=2^{n^{2}}
$$

where $\mathcal{U} \mathcal{A}$ is the set of $2 n \times n$ UASMs and neg $(U A)$ is the number of -1 's in $U A$. This result was conjectured by Propp [14] and proved by Eisenkölbl [2] and independently by Chapman. It is also derivable from Kuperberg [9]. More generally, we show that

$$
\sum_{U A \in \mathcal{U} \mathcal{A}} t^{\operatorname{ssi}(U A)+\operatorname{bar}(U A)}(1+t)^{\operatorname{neg}(U A)}=(1+t)^{n^{2}},
$$

where $\operatorname{ssi}(U A)$ and $\operatorname{bar}(U A)$ are parameters, defined below, associated with each $U A \in \mathcal{U} \mathcal{A}$. More generally still, we show that

$$
D_{s p(2 n)}(x ; t) s p_{\lambda}(x ; t)=\sum_{U A \in \mathcal{U} \mathcal{A}^{\lambda+\delta}(2 n)} t^{\operatorname{ssi}(U A)+\operatorname{bar}(U A)}(1+t)^{\operatorname{neg}(U A)} x^{\operatorname{wgt}(U A)},
$$

where $s p_{\lambda}(x ; t)$ is a $t$-deformation of the character of the irreducible representation of $s p(2 n)$ specified by the partition $\lambda$ and $D_{s p(2 n)}(x ; t)$ is a corresponding $t$-deformation of the denominator formula in the case of $\operatorname{sp}(2 n)$. In this formula (1.3) each indeterminate $x_{i}$ can be thought of as a formal exponential $e^{\epsilon_{i}}$, where the $\epsilon_{i}$ for $i=1,2, \ldots, n$ form a basis of the weight space of $s p(2 n)$. It follows that the $x$-weighting signified by each contribution $x^{\operatorname{wgt}(U A)}=$ $x^{\beta}=x_{1}^{\beta_{1}} x_{2}^{\beta_{2}} \cdots x_{n}^{\beta_{n}}$ serves to define a weight vector $\beta=\beta_{1} \epsilon_{1}+\beta_{2} \epsilon_{2}+\cdots+\beta_{n} \epsilon_{n}$ of $\operatorname{sp}(2 n)$.

The organisation of the paper is such that alternating sign matrices and their U-turn manifestations are introduced in Section 2 and symplectic shifted tableaux in Section 3. It is pointed out that the latter may be viewed as being constructed from a sequence of ribbon strips. It is this structure which is exploited in Section 4 to prove the bijective nature of a map, $\Psi$, from each $s p(2 n)$-standard shifted tableau $S T$ of shape specified by a partition $\mu$, all of whose parts are distinct, to a $2 n \times m \mu$-UASM $U A=\Psi(S T)$, where $m=\mu_{1}$, the largest part of $\mu$.

As a precursor to invoking two independent types of weighting of both $\mu$-UASMs and symplectic shifted tableaux, square ice graphs and the corresponding square ice configuration matrices are introduced in Section 5. These configuration matrices then provide a natural way to motivate and describe two different types of weighting of $\mu$-UASMs, namely an $x$-weighting and a $t$-weighting. Corresponding weightings are then provided for symplectic shifted tableaux. As indicated above these latter weightings are those known to be relevant both to the character theory of $\operatorname{sp}(2 n)[5,6]$ and to the deformation of Weyl's denominator formula for $s p(2 n)$ [4]. This is then exploited in Section 6 to provide a set of variously weighted enumeration formulae for $\mu$-UASMs, symplectic shifted tableaux and square ice configuration matrices. 


\section{Alternating sign matrices}

Alternating sign matrices, ASMs, are square matrices all of whose elements are 0,1 or -1 , such that the first and last non-zero entries of each row and column are 1's and the non-zero entries within each row and column alternate in sign. See, for example, the $4 \times 4$ ASM $A$ in equation (2.1). Here and elsewhere we use $\overline{1}$ to denote -1 .

$$
A=\left[\begin{array}{llll}
0 & 1 & 0 & 0 \\
1 & \overline{1} & 0 & 1 \\
0 & 0 & 1 & 0 \\
0 & 1 & 0 & 0
\end{array}\right]
$$

The number, $A(m)$, of $m \times m$ ASMs is described by the famous formula:

$$
A(m)=\prod_{j=0}^{m-1} \frac{(3 j+1) !}{(m+j) !}
$$

The first proof of this formula was given by Zeilberger [22]. A second proof is due to Kuperberg [8], and a complete history is to be found in Bressoud [1].

From the outset of this theory of ASMs it was found convenient by Mills, Robbins and Rumsey [12] to count them according to the position $k$ of the single 1 that necessarily appears in the top row of each ASM. Deleting the top row of such an ASM gives a generalisation of an ASM in the form of an $(m-1) \times m$ matrix in which the row sums are all 1 , but the column sum is 0 for the $k$ th column and 1 for the others. More generally, one encounters ASMs with more than one column having sum 0 . We follow the terminology of Okada [13] who generalized ASMs by defining a set of $n \times m \mu$-alternating sign matrices, $\mu$-ASMs, associated with each partition $\mu=\left(\mu_{1}, \mu_{2}, \ldots, \mu_{n}\right)$ whose parts $\mu_{j}$ for $j=1,2, \ldots, n$ are all distinct and positive. These $\mu$-ASMs have properties similar to ordinary ASMs, but have column sums 1 only in those columns indexed by $q=\mu_{j}$ for some $j$ and have column sums 0 in all the other columns indexed by $q \neq \mu_{j}$ for any $j$. More formally, for each partition $\mu$ of length $\ell(\mu)=n$, all of whose parts are distinct, and for which $\mu_{1} \leq m$, an $n \times m$ matrix $A=\left(a_{i q}\right)$ belongs to the set $\mathcal{A}^{\mu}(n)$ of $n \times m \mu$-ASMs if the following conditions are satisfied:

$$
\begin{array}{lll}
\text { (O1) } & a_{i q} \in\{-1,0,1\} & \text { for } 1 \leq i \leq n, 1 \leq q \leq m ; \\
\text { (O2) } & \sum_{q=p}^{m} a_{i q} \in\{0,1\} & \text { for } 1 \leq i \leq n, 1 \leq p \leq m ; \\
\text { (O3) } & \sum_{i=j}^{n} a_{i q} \in\{0,1\} & \text { for } 1 \leq j \leq n, 1 \leq q \leq m ; \\
\text { (O4) } & \sum_{q=1}^{m} a_{i q}=1 & \text { for } 1 \leq i \leq n ; \\
\text { (O5) } & \sum_{i=1}^{n} a_{i q}=\left\{\begin{array}{lll}
1 & \text { if } q=\mu_{k} \text { for some } k \\
0 & \text { otherwise } & \text { for } 1 \leq q \leq m, 1 \leq k \leq n .
\end{array}\right.
\end{array}
$$


The alternating sign matrices with a U-turn boundary, UASMs, are a variation on ordinary ASMs developed by Kuperberg [9] after a paper of Tsuchiya [19]. UASMs have an even number of rows. Each column of a UASM is of the same form as that of an ordinary ASM. Each successive pair of rows of a UASM reading first from right to left across the top row of the pair and then from left to right across the bottom row of the pair is like a row of an ASM. Typically we have the $6 \times 3$ UASM $U A$

$$
U A=\left[\begin{array}{lll}
1 & 0 & 0 \\
\overline{1} & 1 & 0 \\
0 & \overline{1} & 1 \\
1 & 0 & 0 \\
0 & 1 & 0 \\
0 & 0 & 0
\end{array}\right]
$$

The number, $A_{U}(2 n)$, of UASMs of size $2 n \times n$ is [9]

$$
A_{U}(2 n)=2^{n}(-3)^{n^{2}} \prod_{\substack{1 \leq i \leq 2 n+1 \\ 1 \leq k \leq n}} \frac{1+6 k-3 i}{2 n+1+2 k-i}
$$

Alternatively, thanks to their connection with vertically symmetric ASMs (VSASMs) or flip symmetric ASMs (FSASMs), and a recurrence relation for the number of the latter due to Robbins [16], we have

$$
A_{U}(2 n)=A_{U}(2 n-2)\left(\begin{array}{c}
6 n-2 \\
2 n
\end{array}\right) /\left(\begin{array}{c}
4 n-2 \\
2 n
\end{array}\right)
$$

with $A_{U}(2)=2$. In either case we obtain:

$$
\begin{array}{cccccccc}
n & 1 & 2 & 3 & 4 & 5 & 6 & \cdots \\
A_{U}(2 n) & 2 & 2^{2} \cdot 3 & 2^{3} \cdot 26 & 2^{4} \cdot 646 & 2^{5} \cdot 45885 & 2^{6} \cdot 9304650 & \cdots
\end{array}
$$

Here we extend UASMs to the case of $\mu$-alternating sign matrices with a U-turn boundary. These were first defined in Hamel and King [4] in the context of deformations of Weyl's denominator formula for characters of the symplectic Lie algebra $s p(2 n)$ and were called $s p(2 n)$-generalised alternating sign matrices. It is this connection with characters of $s p(2 n)$ and the denominator formula which will allow us to evaluate various weighted sums of $\mu$-UASMs.

Definition 2.1 Let $\mu$ be a partition of length $\ell(\mu)=n$, all of whose parts are distinct, and for which $\mu_{1} \leq m$. Then the matrix $U A=\left(a_{i q}\right)$ is said to belong to the $\operatorname{set} \mathcal{U} \mathcal{A}^{\mu}(2 n)$ of $\mu$-alternating sign matrices with a U-turn boundary if it is a $2 n \times m$ matrix whose elements 
$a_{i q}$ satisfy the conditions:

$$
\begin{aligned}
& \text { (UA1) } a_{i q} \in\{-1,0,1\} \quad \text { for } 1 \leq i \leq 2 n, 1 \leq q \leq m \text {; } \\
& \text { (UA2) } \sum_{q=p}^{m} a_{i q} \in\{0,1\} \quad \text { for } 1 \leq i \leq 2 n, 1 \leq p \leq m \text {; } \\
& \text { (UA3) } \sum_{i=j}^{2 n} a_{i q} \in\{0,1\} \quad \text { for } 1 \leq j \leq 2 n, 1 \leq q \leq m \text {. } \\
& \text { (UA4) } \quad \sum_{q=1}^{m}\left(a_{2 i-1, q}+a_{2 i, q}\right)=1 \quad \text { for } 1 \leq i \leq n ; \\
& \text { (UA5) } \sum_{i=1}^{2 n} a_{i q}=\left\{\begin{array}{ll}
1 & \text { if } q=\mu_{k} \text { for some } k \\
0 & \text { otherwise }
\end{array} \text { for } 1 \leq q \leq m, 1 \leq k \leq n .\right.
\end{aligned}
$$

In the case for which $\mu=\delta=(n, n-1, \ldots, 1)$ and $m=n$, for which (UA5) becomes $\sum_{i=1}^{2 n} a_{i q}=1$ for $1 \leq q \leq n$, this definition is such that the set $\mathcal{U} \mathcal{A}^{\delta}(2 n)$ coincides with the set of U-turn alternating sign matrices, UASMs, defined by Kuperberg [9]. The more general case is exemplified for the partition $\mu=(9,7,6,2,1)$ and $n=5$ by:

$$
U A=\left[\begin{array}{lllllllll}
0 & 0 & 0 & 0 & 0 & 0 & 0 & \overline{1} & 1 \\
1 & 0 & 0 & 0 & 0 & 0 & 0 & 0 & 0 \\
0 & 0 & 0 & 0 & 0 & 0 & 0 & 1 & 0 \\
\overline{1} & 1 & 0 & \overline{1} & 0 & 0 & 1 & 0 & 0 \\
1 & 0 & \overline{1} & 1 & 0 & 0 & 0 & 0 & 0 \\
0 & 0 & 0 & \overline{1} & 0 & 1 & 0 & 0 & 0 \\
0 & \overline{1} & 0 & 1 & 0 & 0 & 0 & 0 & 0 \\
0 & 0 & 1 & 0 & 0 & 0 & 0 & 0 & 0 \\
\overline{1} & 1 & 0 & 0 & 0 & 0 & 0 & 0 & 0 \\
1 & 0 & 0 & 0 & 0 & 0 & 0 & 0 & 0
\end{array}\right] \in \mathcal{U} \mathcal{A}^{\mu}(2 n)
$$

As can be seen the successive column sums reading from right to left are 1, 1, 0, 0, 0, 1, 1, 0,1 , with the 1 's appearing in columns $1,2,6,7,9$ specifying the parts of $\mu$. The individual row sums reading from top to bottom are $0,1,1,0,1,0,0,1,0,1$ so that all the $U$-turn row sums for consecutive pairs of rows are 1 , as required.

In the proof of the bijection between $\mu$-UASMs and symplectic shifted tableaux in Section 4 it will be useful to refine the matrix $U A$. Any $\mu$-UASM $U A$ contains two types of zeros: zeros for which there is a nearest non-zero element to the right in the same row taking the value 1 (positive zeros), and all other zeros (negative zeros). We can then define a map $\phi$ from the matrix $U A$ to a signature matrix $\phi(U A)$, replacing positive zeros and positive ones with plus signs, and negative zeros and negative ones with minus signs. It should be noted that there is no ambiguity in determining which zeros are positive and which are 
negative, so that for each $\mu$-UASM $U A$ the signature matrix $\phi(U A)$ is unique. Moreover, to recover $U A$ from $\phi(U A)$ by means of the inverse map $\phi^{-1}$ it is only necessary in each row to replace each right-most + in a continuous sequence of +'s by 1 and all others +'s by 0 , and the right-most - of any continuous sequence of -'s by -1 , provided that its immediate right-hand neighbour is + , and all other -'s by 0 . This is illustrated in the case of our example (2.9) by

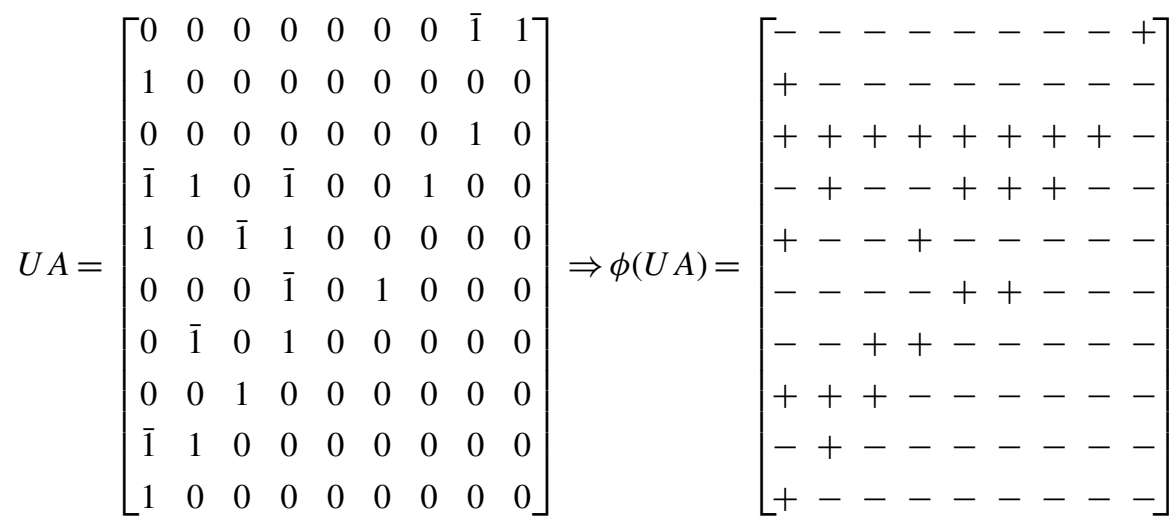

\section{Symplectic shifted tableaux}

Symplectic shifted tableaux are variations on ordinary tableaux and were first introduced in [4] in the context of a symplectic version of Tokuyama's formula [18] for the $t$-deformation of Weyl's denominator formula. A partition $\mu=\left(\mu_{1}, \mu_{2}, \ldots, \mu_{n}\right)$ is a weakly decreasing sequence of non-negative integers. The weight, $|\mu|$, of the partition $\mu$ is the sum of its parts, and its length, $\ell(\mu) \leq n$, is the number of its non-zero parts. Now suppose all of the parts of $\mu$ are distinct. Define a shifted Young diagram $S F^{\mu}$ to be a set of $|\mu|$ boxes arranged in $\ell(\mu)$ rows of lengths $\mu_{i}$ that are left-adjusted to a diagonal line. More formally, $S F^{\mu}=\left\{(i, j) \mid 1 \leq i \leq \ell(\mu), i \leq j \leq \mu_{i}+i-1\right\}$.

For example, for $\mu=(9,7,6,2,1)$ we have

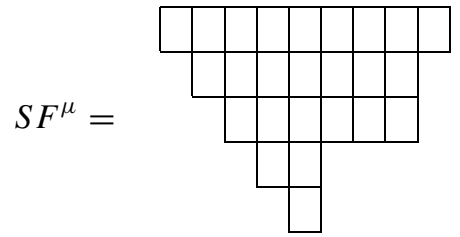

It should be noted that the parts of the partition $\mu^{\prime}=\left(\mu_{1}^{\prime}, \mu_{2}^{\prime}, \ldots, \mu_{m}^{\prime}\right)$, with $m=\mu_{1}$, which is conjugate to $\mu$ specify the lengths of successive diagonals of $S F^{\mu}$. In the above example, $\mu^{\prime}=(5,4,3,3,3,3,2,1,1)$. Quite generally, if all the parts of $\mu$ are distinct, it 
follows that successive parts of $\mu^{\prime}$ differ by at most 1 . In fact, in such a case we have

$$
\mu_{q+1}^{\prime}= \begin{cases}\mu_{q}^{\prime}-1 & \text { if } q=\mu_{k} \text { for some } k \\ \mu_{q}^{\prime} & \text { otherwise }\end{cases}
$$

Each symplectic shifted tableau, $S T$, is the result of filling the boxes of $S F^{\mu}$ with integers from 1 to $n$ and $\overline{1}$ to $\bar{n}$, ordered $\overline{1}<1<\overline{2}<2<\cdots<\bar{n}<n$, subject to a number of restrictions. We require a few more definitions. The profile of a shifted tableau is the sequence of entries on the main diagonal of the shifted tableau. Let $A$ be a totally ordered set, or alphabet, and let $A^{r}$ be the set of all sequences $a=\left(a_{1}, a_{2}, \ldots, a_{r}\right)$ of elements of $A$ of length $r$. Then the general set $\mathcal{S} \mathcal{T}^{\mu}(A ; a)$ is defined to be the set of all standard shifted tableaux, $S T$, with respect to $A$, of profile $a$ and shape $\mu$, formed by placing an entry from $A$ in each of the boxes of $S F^{\mu}$ in such that the following five properties hold:

$$
\begin{array}{lll}
\text { (S1) } & \eta_{i j} \in A & \text { for all }(i, j) \in S F^{\mu} \\
\text { (S2) } & \eta_{i i}=a_{i} \in A & \text { for all }(i, i) \in S F^{\mu} \\
\text { (S3) } & \eta_{i j} \leq \eta_{i, j+1} & \text { for all }(i, j),(i, j+1) \in S F^{\mu} \\
\text { (S4) } & \eta_{i j} \leq \eta_{i+1, j} & \text { for all }(i, j),(i+1, j) \in S F^{\mu} \\
\text { (S5) } & \eta_{i j}<\eta_{i+1, j+1} & \text { for all }(i, j),(i+1, j+1) \in S F^{\mu} .
\end{array}
$$

Informally, we may describe these tableaux as having shifted shape $\mu$ and as being filled with entries from $A$ with profile $a$ such that the entries are weakly increasing from left to right across each row and from top to bottom down each column, and strictly increasing from top-left to bottom-right along each diagonal.

The set $\mathcal{S} \mathcal{T}^{\mu}(n, \bar{n})$ of symplectic shifted tableaux is a specific instance of $\mathcal{S T}^{\mu}(A ; a)$ given by:

Definition 3.1 Let $\mu=\left(\mu_{1}, \mu_{2}, \ldots, \mu_{n}\right)$ be a partition of length $\ell(\mu)=n$, all of whose parts are distinct, and let $A=[n, \bar{n}]=\{1,2, \ldots, n\} \cup\{\overline{1}, \overline{2}, \ldots, \bar{n}\}$ be subject to the order relations $\overline{1}<1<\overline{2}<2<\cdots<\bar{n}<n$. Then the set of all $\operatorname{sp}(2 n)$-standard shifted tableaux of shape $\mu$ is defined by:

$$
\begin{aligned}
& \mathcal{S T}^{\mu}(n, \bar{n})=\left\{S \in \mathcal{S} \mathcal{T}^{\mu}(A ; a) \mid A=[n, \bar{n}], a \in[n, \bar{n}]^{n} \text { with } a_{i} \in\{i, \bar{i}\}\right. \\
& \quad \text { for } i=1,2, \ldots, n\},
\end{aligned}
$$

where the entries $\eta_{i j}$ of each $\operatorname{sp}(2 n)$-standard shifted tableau $S T$ satisfy the conditions (S1)-(S5) of (3.3).

Continuing the above example with $n=5$ and $\mu=(9,7,6,2,1)$, we have typically

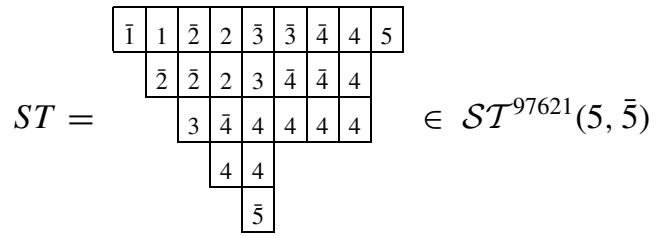


Within each symplectic shifted tableau we can identify a further construct, namely, a ribbon strip [4].

Definition 3.2 The ribbon strips $\operatorname{str}_{k}(S T)$ and $\operatorname{str}_{\bar{k}}(S T)$ consists of all boxes in the symplectic shifted tableau containing $k$ and $\bar{k}$, respectively, with no two such boxes on the same diagonal. Each ribbon strip may consist of one or more continuously connected parts.

By way of example, for $S T$ as in (3.5) $\operatorname{str}_{4}(S T)$ and $\operatorname{str}_{4}(S T)$ take the form

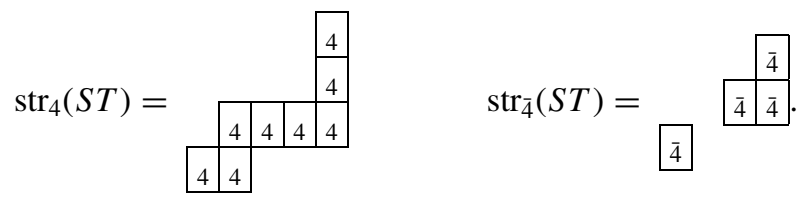

Each symplectic shifted tableaux is nothing other than a collection of ribbon strips nested or wrapped around one another so as to produce a diagram of standard shifted shape. It follows that each $S T \in S \mathcal{T}^{\mu}(n, \bar{n})$ may be encoded by means of a map $\psi$ from $S T$ to a $2 n \times m$ matrix $\psi(S T)$, with $m=\mu_{1}$, in which the rows of $\psi(S T)$, specified by $k$ and $\bar{k}$ taken in reverse order from $n$ at the top to $\overline{1}$ at the bottom, consist of a sequence of symbols + or - in the $q$ th column of $\psi(S T)$, counted from 1 on the left to $m$ on the right, indicating whether or not $\operatorname{str}_{k}(S T)$ and $\operatorname{str}_{\bar{k}}(S T)$, as appropriate, intersects the $q$ th diagonal of $S T$, where diagonals are counted in the north-east direction starting from the main, first diagonal to which the rows of $S T$ are left-adjusted. Typically, applying $\psi$ to our example (3.5) for $S T$ gives $\psi(S T)$ as shown:

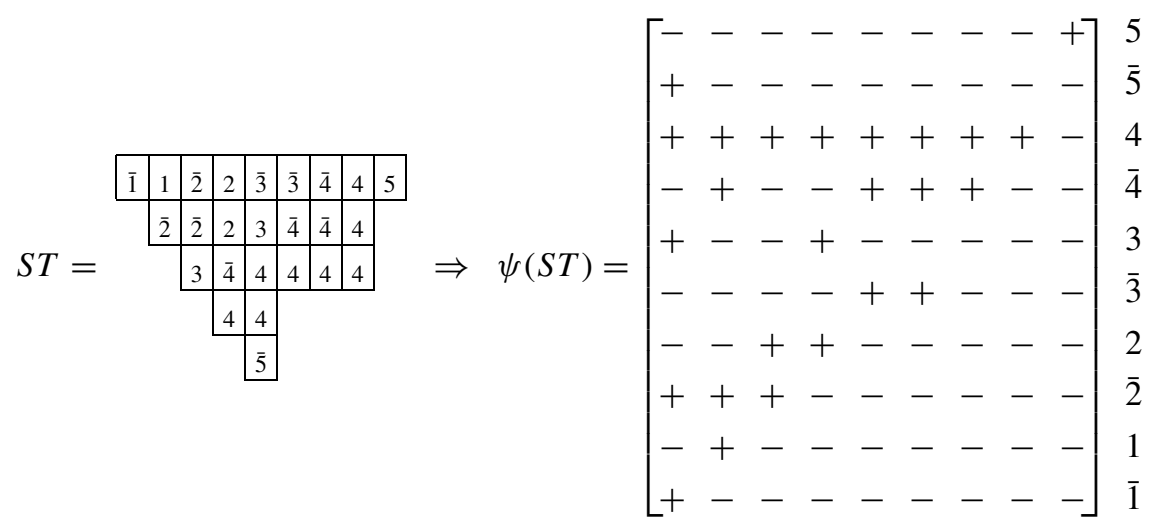

Clearly $\psi(S T)$ is uniquely determined by $S T$ and vice versa. The inverse map $\psi^{-1}$ from $\psi(S T)$ back to $S T$ is accomplished by noting that the elements + in each column of $\psi(S T)$ simply signify by virtue of their row label, $k$ or $\bar{k}$, those entries that appear in the corresponding diagonal of $S T$, arranged in strictly increasing order. 
The strips $\operatorname{str}_{k}(S T)$ and $\operatorname{str}_{\bar{k}}(S T)$, whose connected components are well represented by sequences of consecutive +'s in $\psi(S T)$, play a key role in establishing the bijection between symplectic shifted tableaux and alternating sign matrices with a U-turn boundary.

\section{The bijection}

In Hamel and King [4], we derived a relationship between UASM and symplectic shifted tableaux by first going through monotone triangles. Here we prove the relationship directly. We will find it useful to use the refinement of the UASM defined by $\phi$.

Since the image $\psi(S T)$ of $\psi$ acting on each symplectic shifted tableaux $S T$ is a matrix of \pm 's, the inverse $\phi^{-1}$ may be applied to $\psi(S T)$ to give a matrix of 1's, 1 's and 0 's, which may or may not be a U-turn alternating sign matrix, $U A$. In fact the resulting matrix $\phi^{-1} \circ \psi(S T)$ is always a U-turn alternating sign matrix, and it is shown below in Theorem 4.1 that the map $\Psi=\phi^{-1} \circ \psi$ is a bijective mapping from $\mathcal{S} \mathcal{T}^{\mu}(n, \bar{n})$ to $\mathcal{U} \mathcal{A}^{\mu}(2 n)$.

In the case of our example, the outcome of this procedure mapping from $S T$ to $\psi(S T)$, identifying $\psi(S T)$ with $\phi(U A)$, and then recovering $U A=\phi^{-1} \circ \psi(S T)=\Psi(S T)$ is illustrated by:

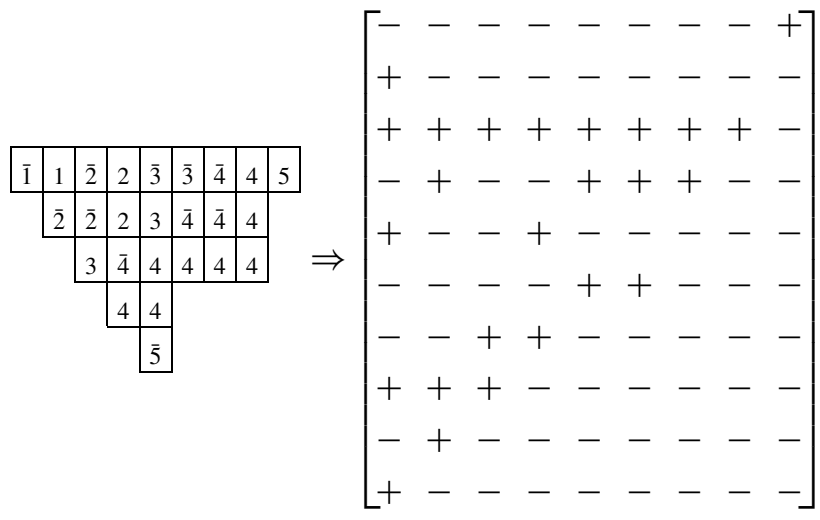

$$
\begin{aligned}
& \Rightarrow\left[\begin{array}{lllllllll}
0 & 0 & 0 & 0 & 0 & 0 & 0 & \overline{1} & 1 \\
1 & 0 & 0 & 0 & 0 & 0 & 0 & 0 & 0 \\
0 & 0 & 0 & 0 & 0 & 0 & 0 & 1 & 0 \\
\overline{1} & 1 & 0 & \overline{1} & 0 & 0 & 1 & 0 & 0 \\
1 & 0 & \overline{1} & 1 & 0 & 0 & 0 & 0 & 0 \\
0 & 0 & 0 & \overline{1} & 0 & 1 & 0 & 0 & 0 \\
0 & \overline{1} & 0 & 1 & 0 & 0 & 0 & 0 & 0 \\
0 & 0 & 1 & 0 & 0 & 0 & 0 & 0 & 0 \\
\overline{1} & 1 & 0 & 0 & 0 & 0 & 0 & 0 & 0 \\
1 & 0 & 0 & 0 & 0 & 0 & 0 & 0 & 0
\end{array}\right]
\end{aligned}
$$


where the rows of the matrices are labelled from top to bottom $n=5, \overline{5}, 4, \overline{4}, 3, \overline{3}, 2, \overline{2}, 1, \overline{1}$, and the columns from left to right $1,2, \ldots, 9=m=\mu_{1}$.

Theorem 4.1 Let $\mu=\left(\mu_{1}, \mu_{2}, \ldots, \mu_{n}\right)$ be a partition of length $\ell(\mu)=n$ whose parts are all distinct. Then the mapping $\Psi=\phi^{-1} \circ \psi$ defines a bijection between the set $\mathcal{S T}^{\mu}(n, \bar{n})$ of $\operatorname{sp}(2 n)$-standard shifted tableaux $S T$ of shape $S F^{\mu}$, and the set $\mathcal{U} \mathcal{A}^{\mu}(2 n)$ of $2 n \times m$ $\mu$-alternating sign matrices $U A$ with a $U$-turn boundary and $m=\mu_{1}$.

Proof: The Definition 3.1 of $\mathcal{S} \mathcal{T}^{\mu}(n, \bar{n})$ ensures that each $s p(2 n)$-standard shifted tableau $S T$ satisfies the properties (S1)-(S5). We need to show, in accordance with the Definition 2.1 of $\mathcal{U} \mathcal{A}^{\mu}(2 n)$, that the properties (UA1)-(UA5) hold for the matrix $U A=\Psi(S T)$ obtained from $S T$ by means of the map $\Psi$.

First, it is obvious from the description of the mappings involved that the only possible matrix elements of $U A$ are $1,-1$, and 0 . Thus (UA1) holds.

Conditions (S3)-(S5) imply that each diagonal of ST contains no repeated entries, leading to the observation that $S T$ consists of a union of ribbon strips as described in Definition 3.2. The map from $S T$ to the matrix $\psi(S T)$ is then such that reading across each row of the matrix $\phi(U A)$ gives sequences of +'s corresponding to each connected component of the relevant ribbon strip. The matrix $\psi(S T)$ is now to be identified with $\phi(U A)$ for some $U A$. The fact that the right-most + of each sequence of consecutive +'s in $\phi(U A)$ is mapped to an element 1 in $U A$, and that the right-most - of each sequence of consecutive -'s is mapped to an element -1 , provided that such a - is followed by a + , means that across each row of the resulting matrix $U A$ we have non-zero entries 1 and -1 that alternate in sign, with the right-most non-zero entry always 1 . This implies the validity of (UA2).

To establish the U-turn nature of $U A$ it is necessary to invoke condition (S2) and the fact that $S T$ is standard only if the entry $\eta_{i i}=a_{i}$ in the $i$ th box of the leading diagonal of $S T$ is either $i$ or $\bar{i}$. The map from $S T$ to $\psi(S T)$ is then such that the elements in the first column of the $i$ th and $i$ th rows are different, one is always + and the other always -. Identifying $\psi(S T)$ with $\phi(U A)$, the first non-zero entries, if they exist, in the corresponding $i$ th and $\bar{i}$ th rows of $U A$ must also differ, one being 1 and the other -1 . This is sufficient to show that the U-turn sequence obtained by reading across the $i$ th row from right to left and then back along the $\bar{i}$ th row from left to right is an alternating sequence of 1's and -1 's. The fact that in both rows the right-most non-zero element must be 1 then ensures the validity of (UA4) since this U-turn alternating sign sequence begins and ends with 1 . If on the other hand either $i$ or $\bar{i}$ is not present in $S T$, then the corresponding row of $\psi(S T)$ will consist wholly of -'s, and identifying $\psi(S T)$ with $\phi(U A)$ leads to the conclusion that the corresponding row of $U A$ consists solely of 0 's, containing no non-zero elements and making no contribution to the U-turn sequence. However, the other row of the pair $i$ and $\bar{i}$ in $\psi(S T)$ must start with a + thereby ensuring that the first non-zero entry in the corresponding row of $U A$ must be 1 . Since the last non-zero element is also 1 , the row sum is 1 and the U-turn condition (UA4) holds yet again.

To deal with (UA3), we consider the diagonals of $S T$. To this end the following schematic diagrams of various portions of the $q$ th and $(q+1)$ th diagonals of $S T$ will prove to 
be helpful.


In these diagrams the labels $i$ and $j$ are the actual entries in the corresponding boxes of $S T$, which may of course be barred or unbarred, while the rules (S3)-(S5) of (3.3) are such that the actual entries of $S T$ in the boxes labeled by $a$ are all distinct, as are those in the boxes labeled by $b$. Moreover, in each case that we will consider each such entry $k$ will necessarily be such that $i<k<j$. We use the notation $n_{a}$ and $n_{b}$ to indicate the number of entries $a$ and $b$, respectively.

All elements 1 in the $q$ th column of the matrix $U A$ constructed from $S T$ by means of the map $\Psi$ correspond to connected components of ribbon strips of $S T$ terminating in the $q$ th diagonal, by virtue of their connection with right-most +'s in continuous sequences of + 's in the rows of $\psi(S T)=\phi(U A)$. Similarly all elements -1 in the $q$ th column of the matrix $U A$ correspond to connected components of ribbon strips starting in the $(q+1)$ th diagonal, by virtue of their connection with the right-most -'s immediately preceding a + in the rows of $\psi(S T)=\phi(U A)$. To see that these non-zero elements in the $q$ th column of $U A$ necessarily alternate in sign, consider two consecutive 1's and the corresponding boxes on the $q$ th diagonal of $S T$. In the schematic diagram $D_{1}$ above, these have been labeled by their entries $i$ and $j$ (which could be barred or unbarred entries). They correspond to the termination of connected components of the $\operatorname{strips}_{\operatorname{str}_{i}}(S T)$ and $\operatorname{str}_{j}(S T)$ in the $q$ th diagonal of $S T$. All $n_{a}$ boxes on the $q$ th diagonal between these $i$ and $j$ boxes, labeled in $D_{1}$ by $a$, must be labeled in $S T$ itself by $n_{a}$ distinct entries $k$ with $i<k<j$. Similarly all $n_{b}$ boxes on the $(q+1)$ th diagonal to the right of $i$ and above $j$, labeled in $D_{1}$ by $b$, must also be labeled in $S T$ by distinct entries $k$ with $i<k<j$. Since $n_{b}=n_{a}+1$ it follows that at least one $b$-label must be distinct from all $a$-labels. If this label is $k$, then a connected component of $\operatorname{str}_{k}(S T)$ must start in the $(q+1)$ th column with no component in the $q$ th column. This leads in the $k$ th row of $\psi(S T)$ to a - followed by a + , and hence to an element -1 in the $q$ th column of $U A$, between the two 1's associated with the boxes $i$ and $j$. Similarly, between any two -1 's in the $q$ th column of $U A$ there must exist an element 1 . The proof is based on the diagram $D_{2}$ above. The details are omitted.

This is not sufficient to prove that (UA3) holds. It is necessary to prove further that the lowest non-zero entry in every column of $U A$ is 1 . The argument is very much as before, this time using the schematic diagram $D_{3}$. In combination with the fact that, as we have proved, the signs of the non-zero elements are alternating in the columns of $U A$, this serves to complete the proof that (UA3) holds. 
The final argument in respect of (UA5) based on the use of the following diagrams is very similar, where the symbol $*$ indicates an empty box to give

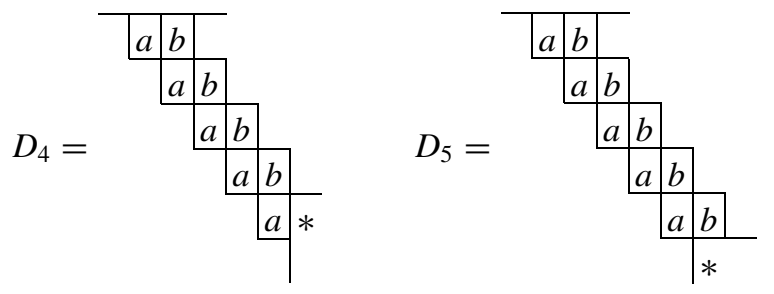

This completes the proof that for all $S T \in \mathcal{S} \mathcal{T}^{\mu}(n, \bar{n})$ we have $U A=\Psi(S T) \in \mathcal{U} \mathcal{A}^{\mu}(2 n)$.

Reversing the argument, Definition 2.1 of $\mathcal{U} \mathcal{A}^{\mu}(2 n)$ ensures that each U-turn alternating sign matrix $U A$ satisfies the properties (UA1)-(UA5). We now need to show that these properties imply, in accordance with the Definition 3.1 of $\mathcal{S} \mathcal{T}^{\mu}(n, \bar{n})$, that $S=\Psi^{-1}(U A)$ satisfies (S1)-(S5), with $A=\{1,2, \ldots, n\} \cup\{\overline{1}, \overline{2}, \ldots, \bar{n}\}$ and $a_{i} \in\{i, \bar{i}\}$ for $i=1,2, \ldots, n$.

First it should be noted that (UA1) guarantees the existence of $\phi(U A)=\psi(S T)$ as a matrix of +'s and -'s. The fact that $U A$ and hence $\psi(S T)$ is $2 n \times m$, with rows labelled by the elements of $A$, then ensures that (S1) holds, since it is the row labels which determine the entries in $S T$.

The U-turn condition embodied in (UA2) and (UA4) then guarantees that each pair of consecutive rows of $\phi(U A)$ counted from the bottom (or top) is such that one of the rows in the pair starts with $\mathrm{a}+$ and the other with a - . In the case of the $i$ th such pair, the row with + in the first column of $\psi(S T)$ determines which one of $i$ or $\bar{i}$ is the leading entry in the $i$ th row of $S T$. This ensures that (S2) holds.

Thereafter, the fact that the entries of $S T$ are built up by adding to the relevant diagonals all the $\overline{1}$ 's, then all the 1 's, followed by all the $\overline{2}$ 's, and so on, ensures that the ordering conditions (S3)-(S5) are automatically satisfied, provided that at every stage, after the addition of all entries $\leq i$, the shape $S F^{\mu(i)}$ of the shifted sub-tableau, $S(i)$, obtained in this way is regular, for all $i=\overline{1}, 1, \overline{2}, \ldots, n$. By regular we mean that the lengths of the rows, left-adjusted as usual to the leading diagonal, are specified by means of a partition, $\mu(i)$, all of whose non-vanishing parts are distinct. Regularity may be proved inductively using once again the diagonal structure of the diagrams.

Hence for all $U A \in \mathcal{U} \mathcal{A}^{\mu}(2 n)$ the conditions (S3)-(S5) apply to $S=\Psi^{-1}(U A)$. Having already established that (S1) and (S2) also apply, we can conclude that for all $U A \in$ $\mathcal{U} \mathcal{A}^{\mu}(2 n)$ we have $S=\Psi^{-1}(U A) \in \mathcal{S T}^{\mu}(n, \bar{n})$.

This completes the proof of Theorem 4 that $\Psi$ provides a bijection between the $s p(2 n)$ standard shifted tableaux $S T \in \mathcal{S T}^{\mu}(n, \bar{n})$ and the U-turn alternating sign matrices $U A \in$ $\mathcal{U} \mathcal{A}^{\mu}(2 n)$ for all partitions $\mu$ of length $\ell(\mu)=n$ whose parts are all distinct.

\section{Square ice}

In order to exploit the above bijection to the full it is necessary to add some $x$ and $t$ dependent weightings to both $U A \in \mathcal{U} \mathcal{A}^{\mu}(2 n)$ and $S T \in \mathcal{S T}^{\mu}(n, \bar{n})$. Although some such 
weightings have already been provided [4], rather similar but not quite identical weightings may perhaps be best motivated and described through the connection between $\mu$-UASMs, symplectic shifted tableaux and the square ice model that has proved to be such an invaluable tool in the study of alternating sign matrices and their enumeration.

Square ice is a two dimensional grid that models the orientation of molecules in frozen water, see for example Lieb [11], Bressoud [1], Lascoux [10]. In frozen water the model is such that each individual molecule, consisting of two hydrogen atoms attached to an oxygen atom, takes up one of the 6 possible orientations (the six vertex model) shown below.

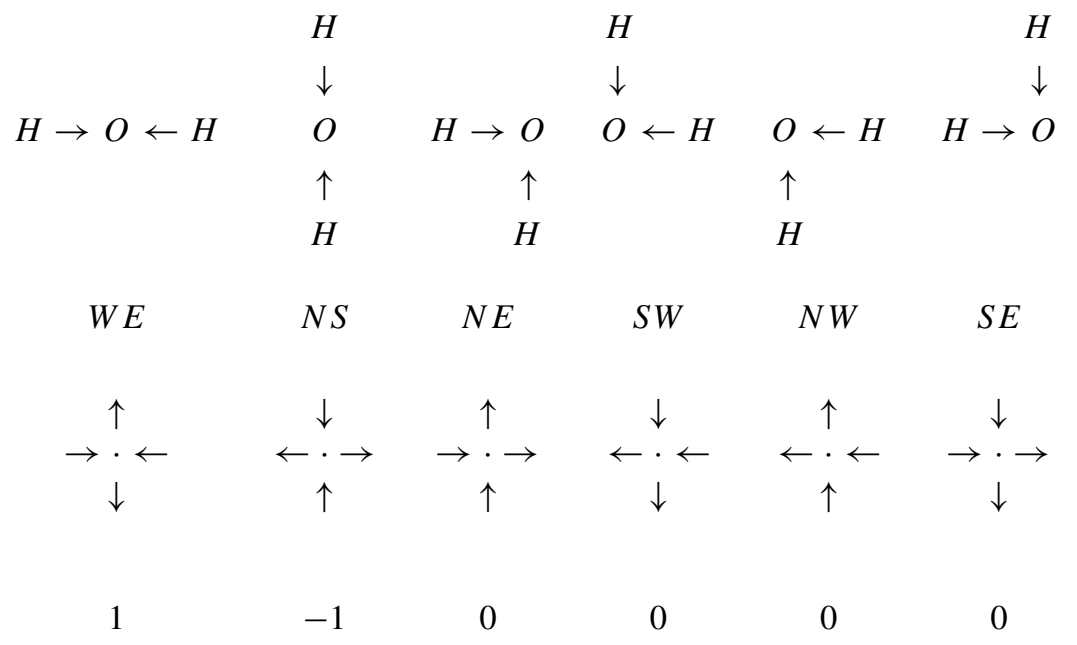

As indicated in the second line of (5.1), the orientation of each molecule may be specified by giving the compass directions of the bonds linking each hydrogen atom to the oxygen atom. Thus WE represents a horizontal molecule, NS a vertical molecule and NE, SW, NW and SE molecules in which the hydrogen bonds are mutually perpendicular. Alternatively, each oxygen atom may be associated with a tetravalent vertex with two incoming and two outgoing edges as shown in the third line of (5.1). At each vertex it is the incoming edges that are associated with the hydrogen bonds displayed in the first line of (5.1).

Square ice configurations [11] consist of arrangements of the above molecules with an oxygen atom at each point of a square $n \times n$ grid. The corresponding square ice graph [11] is one in which the internal vertices sit at the grid points specified by the oxygen atoms. The particular boundary conditions that correspond to ASM were apparently first considered by Korepin [7]. As we have indicated all the internal vertices are tetravalent, with two incoming and two outgoing edges. The boundary vertices, including corner vertices, are not usually drawn. Corner vertices have no edges. Non-corner boundary vertices are of valency one, but there may be boundary conditions on the edges linking them to the internal vertices. Conventionally, each left or right non-corner boundary vertex has an edge pointing towards the adjacent internal vertex, while each top or bottom non-corner boundary vertex has an edge pointing away from the adjacent internal vertex. 
Each such square ice configuration is then associated with an alternating sign matrix. To construct the ASM one merely associates each internal vertex of the type shown in equation (5.1) with the corresponding matrix element $1,-1$ or 0 indicated in the bottom line of (5.1). The fact that the corresponding matrix is an ASM is a consequence of the boundary conditions and the fact that each hydrogen atom is linked to just one oxygen atom. Using this association Kuperberg employed known results on square ice to provide a second proof of the alternating sign matrix conjecture [8].

This natural link between square ice and ordinary ASMs may be generalized slightly so as to account for the U-turns and zero sum columns of our $\mu$-UASMs. It is only necessary to modify the boundary conditions. A zero sum in column $q$ corresponds to a square ice graph with incoming rather than outgoing edges at the top boundary in column $q$. A U-turn corresponds to either an outgoing left boundary edge at row $2 i-1$ and an incoming left boundary edge at row $2 i$, or an incoming left boundary edge at row $2 i-1$ and an outgoing left boundary edge at row $2 i$ as shown in figure 1 . With these changes in boundary conditions we can map the six types of vertices to 1 's, -1 's, and 0 's exactly as before and produce a $\mu$-UASM $U A$. However, the 0's carry less information than is available in the square ice graph. At an intermediate stage in mapping from the square ice graph to a $\mu$-UASM it is helpful to map to a square ice configuration matrix, $C M$, whose matrix elements are just

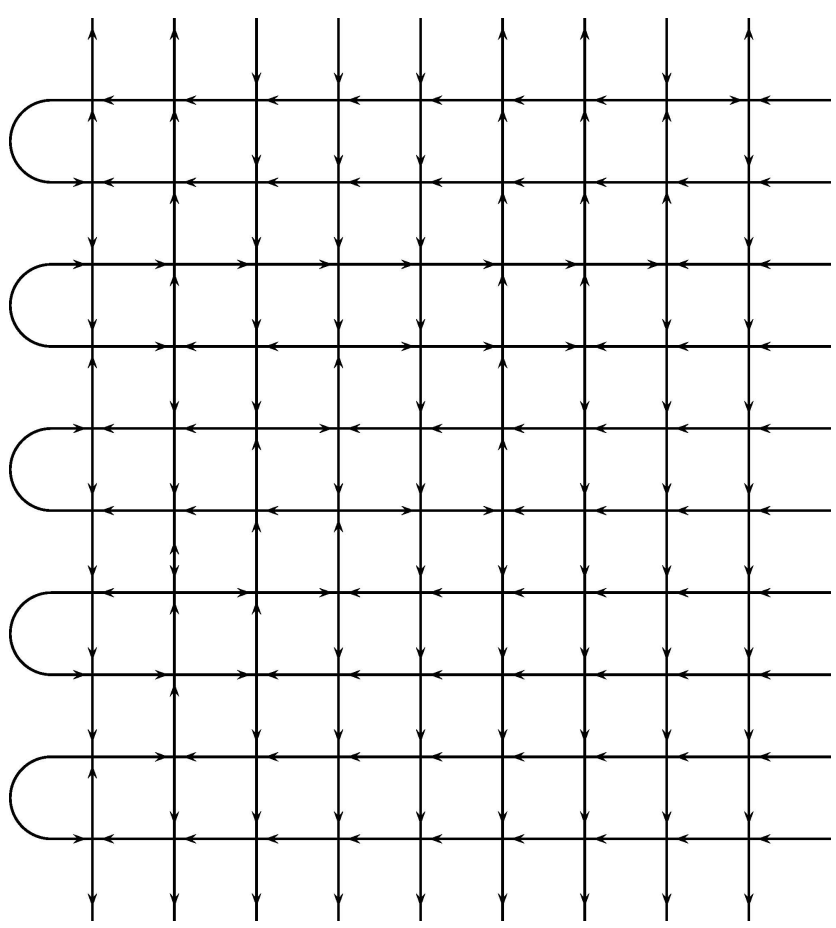

Figure 1. Square ice with U-turn boundary. 
the labels WE, NS, NE, SW, NW, and SE attached to the six types of vertex in (5.1). To be precise, we adopt the following

Definition 5.1 Let $\mu=\left(\mu_{1}, \mu_{2}, \ldots, \mu_{n}\right)$ be a partition of length $\ell(\mu)=n$, all of whose parts are distinct and with largest part $\mu_{1}=m$. Then the configuration matrix $C M$ belongs to the set $\mathcal{C} \mathcal{M}^{\mu}(2 n)$ if it is the image under the map of its vertices to matrix elements in the set $\{W E, N S, N E, S W, N W, S E\}$ defined in (5.1) of a square ice graph on a $2 n \times m$ grid in which each internal vertex has two incoming and two outgoing edges, with all right-hand edges incoming, all bottom edges outgoing, each left-hand pair of edges a U-turn with one edge incoming and one outgoing, and all top edges either outgoing or incoming according as the column number counted from the left is or is not equal to one of the parts of $\mu$.

This is exemplified for the square ice graph of figure 1 by the corresponding configuration matrix $C M$ given in (5.2).

$$
C M=\left[\begin{array}{ccccccccc}
N W & N W & S W & S W & S W & N W & N W & N S & W E \\
W E & N W & S W & S W & S W & N W & N W & N W & S W \\
S E & N E & S E & S E & S E & N E & N E & W E & S W \\
N S & W E & S W & N S & S E & N E & W E & S W & S W \\
W E & S W & N S & W E & S W & N W & S W & S W & S W \\
S W & S W & N W & N S & S E & W E & S W & S W & S W \\
S W & N S & N E & W E & S W & S W & S W & S W & S W \\
S E & N E & W E & S W & S W & S W & S W & S W & S W \\
N S & W E & S W & S W & S W & S W & S W & S W & S W \\
W E & S W & S W & S W & S W & S W & S W & S W & S W
\end{array}\right]
$$

The map $\chi$ from this configuration matrix $C M$ to the corresponding $\mu$-UASM $U A=$ $\chi(C M)$ is then accomplished merely by setting WE and NS to 1 and -1 , respectively, and NE, SW, NW and SE all to 0. The example has been chosen so that the result is the matrix $U A$ appearing in (2.9). It is not difficult to see that for all configuration matrices $C M \in \mathcal{C M}^{\mu}(2 n)$ we have $\chi(C M) \in \mathcal{U} \mathcal{A}^{\mu}(2 n)$. Moreover, $\chi$ is a bijection. The inverse map from $U A \in \mathcal{U}^{\mu}(2 n)$ to $C M=\chi^{-1}(U A) \in \mathcal{C M}^{\mu}(2 n)$ is such that the image under $\chi^{-1}$ of each matrix element 1 and -1 of $U A$ is just WE and NS, respectively. The images of the 0's are NE, SW, NW and SE according as their nearest non-zero neighbours to the right and below are $(1,1),(\overline{1}, \overline{1}),(\overline{1}, 1)$ and $(1, \overline{1})$, respectively, where with some abuse of notation $\overline{1}$ is used to signify either -1 or the absence of any non-zero neighbour in the appropriate direction. These assignments are precisely what is required to ensure that there are no ambiguities in the directions of the edges at any vertex and that collectively they are consistent with the U-turn square ice conditions.

It is convenient to let we $(C M), \operatorname{ns}(C M), \operatorname{ne}(C M), \operatorname{sw}(C M), \operatorname{nw}(C M)$, and $\operatorname{se}(C M)$ denote the total number of matrix elements of the configuration matrix $C M$ that are equal 
to WE, NS, NE, SW, NW and SE, respectively, and to refine this with subscripts $k$ and $\bar{k}$ if the count is restricted to the $(2 n+1-2 k)$ th and $(2 n+2-2 k)$ th rows, respectively. In addition we let ne ${ }_{o}(C M)$ and $\mathrm{se}_{e}(C M)$ denote the total number of matrix elements of $C M$ equal to NE in the odd rows counted from the top, and equal to SE in the even rows, and let $\mathrm{wgt}_{e}(C M)$ denote the total number of matrix elements NE, SE and WE in the even rows. Thus

$$
\begin{aligned}
\operatorname{ne}_{o}(C M) & =\sum_{k=1}^{n} \quad \operatorname{ne}_{k}(C M) ; \\
\operatorname{se}_{e}(C M) & =\sum_{k=1}^{n} \operatorname{se}_{\bar{k}}(C M) ; \\
\operatorname{wgt}_{e}(C M) & =\sum_{k=1}^{n} \quad\left(\operatorname{ne}_{\bar{k}}(C M)+\operatorname{se}_{\bar{k}}(C M)+\mathrm{we}_{\bar{k}}(C M)\right) .
\end{aligned}
$$

The significance of these parameters and the fact that $\chi$ defines a bijection from $C M \in$ $\mathcal{C M}^{\mu}(2 n)$ to $U A \in \mathcal{U} \mathcal{A}^{\mu}(2 n)$ is that we may refer to the 0 's of any such $U A=\chi(C M)$ as being NE, SW, NW or SE 0's if under $\chi^{-1}$ they map to NE, SW, NW or SE, respectively. Then, ne $(C M), \operatorname{sw}(C M), \operatorname{nw}(C M)$ and se $(C M)$ denote the numbers of such 0's in $U A=$ $\chi(C M)$. In the same way the number of 1's and -1 's in $U A$ are given by we $(C M)$ and $\mathrm{ns}(C M)$. Thus the configuration matrix $C M=\chi^{-1}(U A)$ is an alternative refinement of $U A$ to that provided by the signature matrix $\phi(U A)$ exemplified in (2.10). In fact the passage from $\phi(U A)$ to $C M$ is effected by replacing the right-most + and right-most - of any sequence of +'s and -'s in $\phi(U A)$ by $W E$ and $N S$, respectively, with the remaining +'s replaced by either $N E$ or $S E$ and the remaining -'s by either $N W$ or $S W$ in accordance with the above rules regarding nearest non-zero neighbours of the corresponding 0's in $U A$.

All this allows us to define various weightings and statistics on both $U A \in \mathcal{U} \mathcal{A}^{\mu}(2 n)$ and $S T \in \mathcal{S} \mathcal{T}^{\mu}(n, \bar{n})$. First we assign an $x$-weighting to each $\mu$-UASM. To this end let $m_{k}(U A)$ and $m_{\bar{k}}(U A)$ be the number of positive zeros and ones in the $k$ th even and the $k$ th odd row of $U A$, respectively, counted upwards from the bottom for $k=1,2, \ldots, n$. Then

$$
x^{\operatorname{wgt}(U A)}=x_{1}^{m_{1}(U A)-m_{\overline{1}}(U A)} x_{2}^{m_{2}(U A)-m_{\overline{2}}(U A)} \cdots x_{n}^{m_{n}(U A)-m_{\bar{n}}(U A)} .
$$

In our running example (2.10) this gives

$$
x^{\operatorname{wgt}(U A)}=x_{1}^{1-1} x_{2}^{2-3} x_{3}^{2-2} x_{4}^{8-4} x_{5}^{1-1}=x_{2}^{-1} x_{4}^{4} .
$$

It should be noted that $m_{k}(U A)$ and $m_{\bar{k}}(U A)$ are just the number of +'s in the $(2 n+1-2 k)$ th and $(2 n+2-2 k)$ th rows of the signature matrix $\phi(U A)$, respectively.

Equivalently, in terms of the configuration matrix $C M=\chi^{-1}(U A)$ we have

$$
\begin{aligned}
& m_{k}(U A)=m_{k}(C M) \quad \text { with } m_{k}(C M)=\mathrm{ne}_{k}(C M)+\mathrm{se}_{k}(C M)+\mathrm{we}_{k}(C M) ; \\
& m_{\bar{k}}(U A)=m_{\bar{k}}(C M) \quad \text { with } m_{\bar{k}}(C M)=\mathrm{ne}_{\bar{k}}(C M)+\mathrm{se}_{\bar{k}}(C M)+\mathrm{we}_{\bar{k}}(C M),
\end{aligned}
$$


for $k=1,2, \ldots, n$. It follows that

$$
x^{\operatorname{wgt}(U A)}=x^{\operatorname{wgt}(C M)} \quad \text { with } \quad x^{\operatorname{wgt}(C M)}=\prod_{k=1}^{n} x_{k}^{m_{k}(C M)-m_{\bar{k}}(C M)} .
$$

There also exists a standard $x$-weighting of the $s p(2 n)$-symplectic shifted tableaux $S T$. To each entry $k$ or $\bar{k}$ in $S T$ we associate a factor $x_{k}$ or $x_{k}^{-1}$. The product of all these factors for $k=1,2, \ldots, n$ serves to define, as in [4], the $x$-weight of $S T$. Setting $m_{k}(S T)$ and $m_{\bar{k}}(S T)$ equal to the number of entries $k$ and $\bar{k}$, respectively, in $S T$ for $k=1,2 \ldots, n$ we have

$$
x^{\operatorname{wgt}(S T)}=x_{1}^{m_{1}(S T)-m_{\overline{1}}(S T)} x_{2}^{m_{2}(S T)-m_{\overline{2}}(S T)} \cdots x_{n}^{m_{n}(S T)-m_{\bar{n}}(S T)} .
$$

In the example (3.5) this gives

$$
x^{\mathrm{wgt}(S T)}=x_{1}^{1-1} x_{2}^{2-3} x_{3}^{2-2} x_{4}^{8-4} x_{5}^{1-1}=x_{2}^{-1} x_{4}^{4} \text {. }
$$

As can be seen from the bijective mapping from $S T$ to $U A=\Psi(S T)$ by way of $\psi(S T)=$ $\phi(U A)$, illustrated in (2.10), we have

$$
\begin{array}{ll}
m_{k}(S T)=m_{k}(U A) & \text { for } k=1,2, \ldots, n \\
m_{\bar{k}}(S T)=m_{\bar{k}}(U A) & \text { for } k=1,2, \ldots, n,
\end{array}
$$

and hence

$$
x^{\mathrm{wgt}(S T)}=x^{\mathrm{wgt}(U A)} .
$$

In addition to the above $x$-weightings of both $U A \in \mathcal{U} \mathcal{A}^{\mu}(2 n)$ and $S T \in \mathcal{S T}^{\mu}(n, \bar{n})$, we can also assign $t$-weightings to both $U A$ and $S T$. In dealing with $U A$ we require three statistics based on, but not quite identical to those introduced previously [4]. The first statistic, $\operatorname{neg}(U A)$, is defined to be the number of -1 's appearing in $U A$. The second statistic, $\operatorname{bar}(A)$, is defined to be the total number of positive zeros and ones in the even rows of $U A$ counted from the top. This statistic can be read off most easily from $\phi(U A)$. For the third statistic we need the following:

Definition 5.2 Let $U A$ be a $\mu$-UASM with matrix elements $a_{i q}$ for $1 \leq i \leq 2 n$ and $1 \leq q \leq m$. Then $U A$ is said to have a site of special interest, an ssi, at $(i, q)$ if:

(SS1) $\quad a_{i q}=0$

(SS2) $a_{i r}=1$ with $a_{i p}=0$ for $q<p<r \leq m$;

(SS3) either $i$ is odd and $a_{k q}=1$ with $a_{j q}=0$ for $i<j<k \leq 2 n$, or $i$ is even and $a_{k q}=-1$ with $a_{j q}=0$ for $i<j<k \leq 2 n$, or $i$ is even and $a_{j q}=0$ for $i<j \leq 2 n$. 
More graphically, each ssi is the site of a 0 of $U A$ whose nearest non-zero right hand neighbour is 1 , and whose nearest non-zero neighbour below the site is 1 for a site in an odd row counted from the top and either -1 or non-existent for a site in an even row. With this definition, $\operatorname{ssi}(U A)$ is defined to be the number of sites of special interest in $U A$.

Once again it is perhaps easiest to read off these parameters $\operatorname{neg}(U A), \operatorname{bar}(U A)$ and $\operatorname{ssi}(U A)$ from the corresponding configuration matrix $C M=\chi^{-1}(U A)$. In terms of this matrix we have

$$
\begin{aligned}
\operatorname{neg}(U A) & =\operatorname{ns}(C M)=\sum_{k=1}^{n}\left(\mathrm{~ns}_{k}(C M)+\mathrm{ns}_{\bar{k}}(C M)\right) ; \\
\operatorname{bar}(U A) & =\sum_{k=1}^{n}\left(\mathrm{ne}_{\bar{k}}(C M)+\mathrm{se}_{\bar{k}}(C M)+\mathrm{we}_{\bar{k}}(C M)\right) ; \\
\sin (U A) & =\sum_{k=1}^{n}\left(\mathrm{ne}_{k}(C M)+\mathrm{se}_{\bar{k}}(C M)\right) .
\end{aligned}
$$

In the example of (2.10) we have $\operatorname{neg}(U A)=7, \operatorname{bar}(U A)=11$ and $\operatorname{ssi}(U A)=7$, where the seven sites of special interest are indicated by boldface 0 's in $U A$, and by boldface $N E$ 's and $S E$ 's in $C M=\chi^{-1}(U A)$, as shown below in (5.14).

$$
U A=\left[\begin{array}{lllllllll}
0 & 0 & 0 & 0 & 0 & 0 & 0 & \overline{1} & 1 \\
1 & 0 & 0 & 0 & 0 & 0 & 0 & 0 & 0 \\
0 & \mathbf{0} & 0 & 0 & 0 & \mathbf{0} & \mathbf{0} & 1 & 0 \\
\overline{1} & 1 & 0 & \overline{1} & \mathbf{0} & 0 & 1 & 0 & 0 \\
1 & 0 & \overline{1} & 1 & 0 & 0 & 0 & 0 & 0 \\
0 & 0 & 0 & \overline{1} & \mathbf{0} & 1 & 0 & 0 & 0 \\
0 & \overline{1} & \mathbf{0} & 1 & 0 & 0 & 0 & 0 & 0 \\
\mathbf{0} & 0 & 1 & 0 & 0 & 0 & 0 & 0 & 0 \\
\overline{1} & 1 & 0 & 0 & 0 & 0 & 0 & 0 & 0 \\
1 & 0 & 0 & 0 & 0 & 0 & 0 & 0 & 0
\end{array}\right]
$$


The $t$-weight to be attached to each element of $C M=\chi^{-1}(U A)$ can then be tabulated as follows

$\begin{array}{ccc}\text { element } & \text { odd rows } & \text { even rows } \\ W E & 1 & t \\ N S & 1+t & 1+t \\ N E & t & t \\ S W & 1 & 1 \\ N W & 1 & 1 \\ S E & 1 & t^{2}\end{array}$

Comparison of (5.15) with (5.13) shows that this gives a total $t$-weight of

$$
t^{\operatorname{ssi}(U A)+\operatorname{bar}(U A)}(1+t)^{\operatorname{neg}(U A)} .
$$

Applying (5.15) to (5.14) gives the $t$-weighting

$$
U A:(1+t)^{7} \times\left[\begin{array}{ccccccccc}
1 & 1 & 1 & 1 & 1 & 1 & 1 & \overline{1} & 1 \\
t & 1 & 1 & 1 & 1 & 1 & 1 & 1 & 1 \\
1 & t & 1 & 1 & 1 & t & t & 1 & 1 \\
\overline{1} & t & 1 & \overline{1} & t^{2} & t & t & 1 & 1 \\
1 & 1 & \overline{1} & 1 & 1 & 1 & 1 & 1 & 1 \\
1 & 1 & 1 & \overline{1} & t^{2} & t & 1 & 1 & 1 \\
1 & \overline{1} & t & 1 & 1 & 1 & 1 & 1 & 1 \\
t^{2} & t & t & 1 & 1 & 1 & 1 & 1 & 1 \\
\overline{1} & 1 & 1 & 1 & 1 & 1 & 1 & 1 & 1 \\
t & 1 & 1 & 1 & 1 & 1 & 1 & 1 & 1
\end{array}\right]=t^{18}(1+t)^{7}
$$

Turning now to the $t$-weighting of an $s p(2 n)$-shifted tableau $S T$ it is convenient, in order to match contributions to the $t$-weight of $S T$ more precisely to the above contributions to the $t$-weight of $U A=\Psi(S T)$, to modify slightly our previous $t$-weighting of $s p(2 n)$ standard shifted tableaux [4]. This is done as follows. Each entry $k$ in $S T$ belongs to a ribbon $\operatorname{strip}_{\operatorname{str}_{k}}(S T)$ as in Definition 3.2. The $t$-weight of an entry $k$ is then defined to be $t$ if the entry immediately above this entry is also in $\operatorname{str}_{k}(S T)$, otherwise its $t$-weight is 1 . Similarly the $t$-weight of an entry $\bar{k}$ is defined to be $t^{2}$ if the entry immediately to its right is also in $\operatorname{str}_{\bar{k}}(S T)$, otherwise its $t$-weight is $t$. There is an additional $t$-weighting of $(1+t)$ for every connected component of a strip $\operatorname{str}_{k}(S T)$ or $\operatorname{str}_{\bar{k}}(S T)$ that does not start on the main diagonal. In order to codify this, let $\operatorname{str}(S T)$ be the total number of continuously connected 
components of all $\operatorname{str}_{k}(S T)$ and $\operatorname{str}_{\bar{k}}(S T)$ for $k=1,2, \ldots, n$, and let $\operatorname{bar}(S T)$ be the total number of barred entries in $S T$. In addition let

$$
\operatorname{var}(S T)=\sum_{k=1}^{n}\left(\operatorname{row}_{k}(S T)-\operatorname{con}_{k}(S T)+\operatorname{col}_{\bar{k}}(S T)-\operatorname{con}_{\bar{k}}(S T)\right)
$$

where $\operatorname{row}_{k}(S T)$ and $\operatorname{col}_{k}(S T)$ are the number of rows and columns of $S T$ containing a $k$ and $\bar{k}$, respectively, and $\operatorname{con}_{k}(S T)$ and $\operatorname{con}_{\bar{k}}(S T)$ are the number of continuously connected components of $\operatorname{str}_{k}(S T)$ and $\operatorname{str}_{\bar{k}}(S T)$, respectively. This statistic $\operatorname{var}(S T)$ represents a measure of the upward steps in $\operatorname{all} \operatorname{str}_{k}(S T)$ and the rightward steps in $\operatorname{all}_{\operatorname{str}_{\bar{k}}}(S T)$. In terms of the parameter $\operatorname{hgt}(S T)$ used in [4], we have $\operatorname{var}(S T)=\operatorname{hgt}(S T)+$ $\operatorname{bar}(S T)$.

For the strips of (3.6) this $t$-weighting is illustrated by
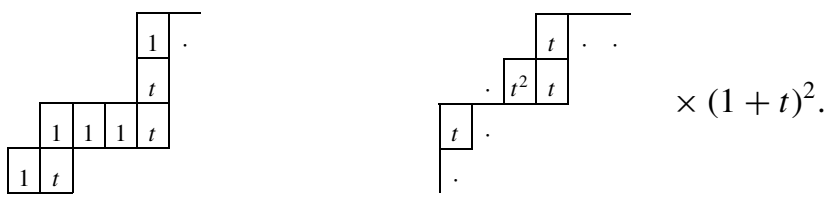

More generally, putting all such strips together we obtain the following $t$-weighting of $S T$ from (3.5):

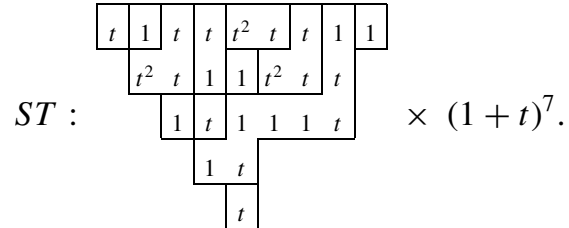

As we have seen the bijection between $S T \in \mathcal{S} \mathcal{T}^{\mu}(n, \bar{n})$ and $U A \in \mathcal{U} \mathcal{A}^{\mu}(2 n)$ is such that the $(2 n+1-2 k)$ th and $(2 n+2-2 k)$ th rows of $U A$ are determined by $\operatorname{str}_{k}(S T)$ and $\operatorname{str}_{\bar{k}}(S T)$, respectively, for $k=1,2, \ldots, n$. It is not difficult to see that each entry $k$ of weight $t$ corresponds to an NE 0 of $U A$, while those of weight 1 correspond either to a SE 0 or to a WE entry 1 if $k$ is the last entry of a connected component of $\operatorname{str}_{k}(S T)$. In the same way each entry $\bar{k}$ of weight $t^{2}$ corresponds to a SE 0 of $U A$, while those of weight $t$ correspond either to a NE 0 or to a WE entry 1 if $\bar{k}$ is the last entry of a connected component of $\operatorname{str}_{k}(S T)$. The additional weighting factors $(1+t)$ are associated with the NS -1 's of $U A$ since it is these -1 's that signal the start of a sequence of positive 0 's ending in a 1 . In terms of the elements of the corresponding configuration matrix, $C M=\chi^{-1}(U A)$, arising 
from the square ice model we have

$$
\begin{aligned}
& \operatorname{str}(S T)=\operatorname{ns}(C M)=\sum_{k=1}^{n}\left(\mathrm{~ns}_{k}(C M)+\mathrm{ns}_{\bar{k}}(C M)\right) \\
& \operatorname{bar}(S T)=\sum_{k=1}^{n}\left(\operatorname{ne}_{\bar{k}}(C M)+\mathrm{se}_{\bar{k}}(C M)+\mathrm{we}_{\bar{k}}(C M)\right) \\
& \operatorname{var}(S T)=\sum_{k=1}^{n}\left(\operatorname{ne}_{k}(C M)+\operatorname{se}_{\bar{k}}(C M)\right)
\end{aligned}
$$

It follows from (5.13) that for $U A=\Psi(S T)$ we have

$$
\operatorname{neg}(U A)=\operatorname{str}(S T)-n, \quad \operatorname{bar}(U A)=\operatorname{bar}(S T), \quad \operatorname{ssi}(U A)=\operatorname{var}(S T) .
$$

The coincidence of the $t$-weighting of $S T$ and $U A$ is exemplified in the case of our running example by

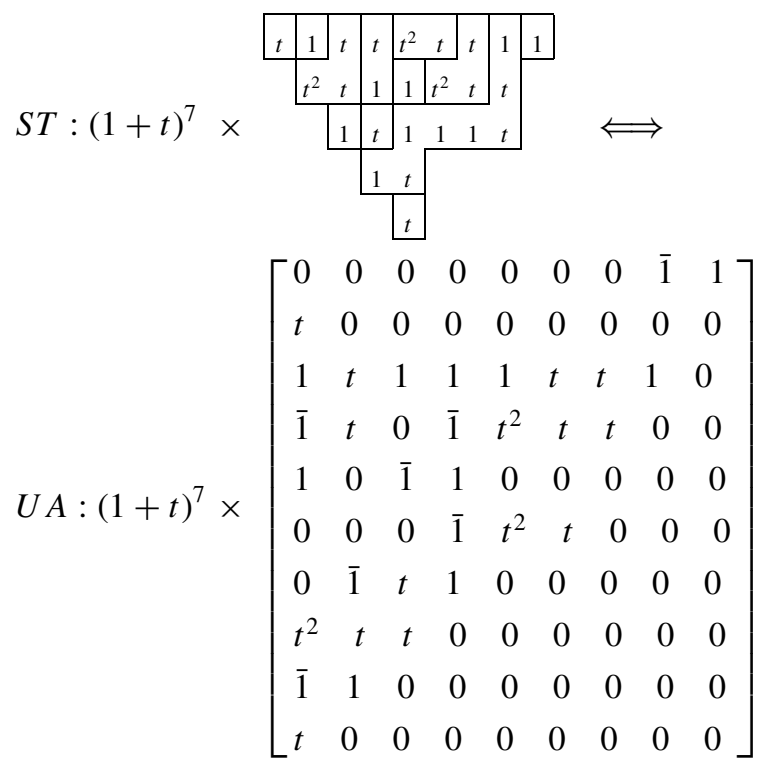

where, in particular, the 3rd and 4th rows of the $t$-weighting of $U A$ are obtained from the $t$-weighting of $\operatorname{str}_{4}(S T)$ and $\operatorname{str}_{4}(S T)$ displayed in (5.20). In contrast to (5.17) the NW and SW 0's of $U A$ have been mapped to 0 to indicate that they have no counterpart in $S T$. In fact they correspond to the diagonals of $S T$ on which the relevant strips have no box, as indicated for example by the 's in (5.19). Ignoring these 0's, the corresponding $t$-weight of both $S T$ and $U A$ is the product of all the displayed powers of $t$ together with the seven factors $(1+t)$ arising from the seven continuously connected components of the strips of $S T$ that do not start on the main diagonal, and equivalently from the seven $\overline{1}$ 's of $U A$. It 
should be noted that the sites of special interest in $U A$ correspond to the location of the $t$ 's and $t^{2}$ 's in the odd and even rows, respectively, of the $t$-weighted version of $U A$.

It is perhaps worth summarising the $x$ and $t$-weighting by pointing out that in terms of the labelling used in $C M=\chi^{-1}(U A)$ the combined $x$ and $t$-weighting translates to

$$
\begin{cases}(1+t)^{\mathrm{ns}(C M)} t^{\mathrm{ne}(C M)}\left(x_{k}\right)^{\mathrm{ne}(C M)+\operatorname{se}(C M)+\mathrm{we}(C M)} & \text { for row } 2 n+1-2 k \\ (1+t)^{\mathrm{ns}(C M)} t^{\mathrm{se}(C M)}\left(t x_{k}^{-1}\right)^{\mathrm{ne}(C M)+\operatorname{se}(C M)+\mathrm{we}(C M)} & \text { for row } 2 n+2-2 k\end{cases}
$$

for $k=1,2, \ldots, n$.

\section{Weighted enumeration}

Propp [15] has provided data for and made a number of conjectures about the weighted enumeration of UASMs. Eisenkölbl [2] has proved a number of these conjectures, and a number are derivable from Kuperberg [9]. Here we delineate a new family of weighted enumerations of UASMs, and more generally of $\mu$-UASMs through their connection with symplectic shifted tableaux and show their overlap with the results of Propp.

To do this in the greatest generality, we shall also need the notion of ordinary symplectic tableaux $[5,6,17]$. Let $\lambda=\left(\lambda_{1}, \lambda_{2}, \ldots, \lambda_{r}\right)$ be a partition of length $\ell(\lambda)=r \leq n$ and weight $|\lambda|$. Each such partition specifies a Young diagram $F^{\lambda}$ consisting of $|\lambda|$ boxes arranged in $\ell(\lambda)$ rows of length $\lambda_{i}$ that are left adjusted to a vertical line. For example, for $\lambda=(4,3,3)$ we have

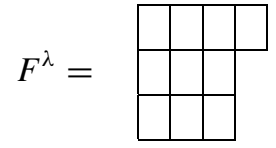

Each symplectic tableau, $T$, of shape $\lambda$ is then the result of filling the boxes of $F^{\lambda}$ with integers from 1 to $n$ and $\overline{1}$ to $\bar{n}$, ordered $\overline{1}<1<\overline{2}<2<\cdots<\bar{n}<n$, subject to a number of restrictions. This time let the profile of a tableau be the sequence of entries obtained by reading down the first, left-most column. Let $A$ be a totally ordered set, or alphabet, and let $A^{r}$ be the set of all sequences $a=\left(a_{1}, a_{2}, \ldots, a_{r}\right)$ of elements of $A$ of length $r$. Then the general set $\mathcal{T}^{\lambda}(A ; a)$ is defined to be the set of all standard shifted tableaux, $S T$, with respect to $A$, of profile $a$ and shape $\lambda$, formed by placing an entry from $A$ in each of the boxes of $F^{\lambda}$ in such that the following four properties hold:

$$
\begin{array}{lll}
\text { (T1) } & \eta_{i j} \in A & \text { for all }(i, j) \in F^{\lambda} \\
\text { (T2) } & \eta_{i i}=a_{i} \in A & \text { for all }(i, 1) \in F^{\lambda} \\
\text { (T3) } & \eta_{i j} \leq \eta_{i, j+1} & \text { for all }(i, j),(i, j+1) \in F^{\lambda} \\
\text { (T4) } & \eta_{i j}<\eta_{i+1, j} & \text { for all }(i, j),(i+1, j) \in F^{\lambda}
\end{array}
$$

These tableaux of shape $\lambda$ and profile $a$ have entries from $A$ that are weakly increasing from left to right across each row and are strictly increasing from top to bottom down each column. 
The set $\mathcal{T}^{\lambda}(s p(2 n))$ of all $s p(2 n)$-standard tableaux of shape $\lambda$ is a specific instance of $\mathcal{T}^{\lambda}(A ; a)$ given by

Definition 6.1 Let $\lambda=\left(\lambda_{1}, \lambda_{2}, \ldots, \lambda_{r}\right)$ be a partition of length $\ell(\mu)=r \leq n$, and let $A=[n, \bar{n}]=\{1,2, \ldots, n\} \cup\{\overline{1}, \overline{2}, \ldots, \bar{n}\}$ be subject to the order relations $\overline{1}<1<\overline{2}<$ $2<\cdots<\bar{n}<n$. Then the set of all $\operatorname{sp}(2 n)$-standard tableaux of shape $\lambda$ is defined by:

$$
\mathcal{T}^{\lambda}(s p(2 n))=\left\{T \in \mathcal{T}^{\lambda}(A ; a) \mid A=[n, \bar{n}], a \in[n, \bar{n}]^{r} \text { with } a_{i} \geq i \text { for } i=1,2, \ldots, r\right\},
$$

where the entries $\eta_{i j}$ of each $s p(2 n)$-standard tableau $T$ satisfy the conditions (T1)-(T4) of (6.2).

Typically, for $n=5$ and $\lambda=(4,3,3)$ we have

$$
T=\begin{array}{|l|l|l|l}
\hline \overline{1} & \overline{1} & 1 & 5 \\
\hline 2 & 2 & 4 \\
\hline 4 & \overline{5} & \overline{5} \\
\hline
\end{array} \in \mathcal{T}^{433}(10) .
$$

The symplectic Schur function $[5,6,17]$, which with a suitable interpretation of the indeterminates $x_{i}$ for $i=1,2, \ldots, n$ is the character of the irreducible representation of the Lie algebra $\operatorname{sp}(2 n)$ specified by $\lambda$, then takes the form

$$
s p_{\lambda}(x)=s p_{\lambda}\left(x_{1}, x_{2}, \ldots, x_{n}\right)=\sum_{T \in \mathcal{T}^{\lambda}(s p(2 n))} x^{\mathrm{wgt}(T)},
$$

where the sum is now over all $\operatorname{sp}(2 n)$-standard tableaux $T$ of shape $\lambda$ and

$$
x^{\mathrm{wgt}(T)}=x_{1}^{m_{1}(T)-m_{\overline{1}}(T)} x_{2}^{m_{2}(T)-m_{\overline{2}}(T)} \cdots x_{n}^{m_{n}(T)-m_{\bar{n}}(T)},
$$

with $m_{k}(T)$ and $m_{\bar{k}}(T)$ equal to the number of entries $k$ and $\bar{k}$, respectively, in $T$.

It is useful in the present context to generalise this by introducing some $t$-dependence and defining

$$
s p_{\lambda}(x ; t)=\sum_{T \in \mathcal{T}^{\lambda}(s p(2 n))} t^{2 \operatorname{bar}(T)} x^{\operatorname{wgt}(T)}
$$

where $\operatorname{bar}(T)$ is the number of barred entries in $T$, that is

$$
\operatorname{bar}(T)=\sum_{k=1}^{n} m_{\bar{k}}(T)
$$

In our example (6.4) we have $\operatorname{bar}(T)=4$ and

$$
x^{\mathrm{wgt}(T)}=x_{1}^{1-2} x_{2}^{3-0} x_{3}^{0-0} x_{4}^{2-0} x_{5}^{0-2}=x_{1}^{-1} x_{2}^{3} x_{4}^{2} x_{5}^{-2} .
$$


In this context the $t$-deformation of the denominator of Weyl's character formula for $\operatorname{sp}(2 n)$ takes the form

$$
D_{s p(2 n)}(x ; t)=\prod_{1 \leq i \leq n} x_{i}^{n-i+1} \prod_{1 \leq i \leq n}\left(1+t x_{i}^{-2}\right) \prod_{1 \leq i<j \leq n}\left(1+t x_{i}^{-1} x_{j}\right)\left(1+t x_{i}^{-1} x_{j}^{-1}\right) .
$$

In our previous paper [4] we derived the following extension to Tokuyama's formula [18] for the expansion of (6.10), namely

Theorem 6.2 Let $\lambda$ be a partition into no more that $n$ parts and let $\delta=(n, n-1, \ldots, 1)$. Then

$$
D_{s p(2 n)}(x ; t) s p_{\lambda}(x ; t)=\sum_{S T \in \mathcal{S} \mathcal{T}^{\lambda+\delta}(n, \bar{n})} t^{\operatorname{var}(S T)+\operatorname{bar}(S T)}(1+t)^{\operatorname{str}(S T)-n} x^{\mathrm{wgt}(S T)},
$$

where the summation is taken over all $s p(2 n)$-standard shifted tableaux $S T$ of shape $\mu=$ $\lambda+\delta$.

Thanks to the bijection between $S T \in \mathcal{S T}^{\mu}(n, \bar{n})$ and $U A \in \mathcal{U} \mathcal{A}^{\mu}(2 n)$ and the equivalence between the $x$ and $t$-weightings of $S T$ and $U A$ this theorem can be recast in terms of $\mu$-UASMs as follows:

Theorem 6.3 Let $\lambda$ be a partition into no more than $n$ parts, let $\delta=(n, n-1, \ldots, 1)$, and let $m=\lambda_{1}+n$. Then

$$
D_{s p(2 n)}(x ; t) s p_{\lambda}(x ; t)=\sum_{U A \in \mathcal{U} \mathcal{A}^{\lambda+\delta}(2 n)} t^{\operatorname{ssi}(U A)+\operatorname{bar}(U A)}(1+t)^{\operatorname{neg}(U A)} x^{\operatorname{wgt}(U A)},
$$

where the summation is taken over all $2 n \times m$ UASMs whose non-vanishing column sums are 1 or 0 according as the column number is or is not a part of $\mu=\lambda+\delta$.

Finally, in terms of the square ice configuration matrices we have

Theorem 6.4 Let $\lambda$ be a partition into no more than $n$ parts, let $\delta=(n, n-1, \ldots, 1)$ and let $m=\lambda_{1}+n$. Then

$$
D_{s p(2 n)}(x ; t) s p_{\lambda}(x ; t)=\sum_{C M \in \mathcal{C M}^{\lambda+\delta}(2 n)} t^{\mathrm{ne}_{o}(C M)+\mathrm{se}_{e}(C M)+\mathrm{wgt}_{e}(C M)}(1+t)^{\mathrm{ns}(C M)} x^{\mathrm{wgt}(C M)},
$$

where the summation is taken over all $2 n \times m U$-turn square ice configuration matrices $C M$ whose top-most element in each column is either NW or NS if the column number is a part of $\mu=\lambda+\delta$ and is $S W$ or WE otherwise. 
By setting $x_{1}=x_{2}=x_{3}=\cdots=x_{n}=1$ in these formulae we derive the following results

Corollary 6.5 Let $\lambda$ be a partition into no more than $n$ parts, let $\delta$ be the partition $(n, n-1, \ldots, 1)$, and let $m=\lambda_{1}+n$. Then

$$
\begin{aligned}
(1+t)^{n^{2}} s p_{\lambda}(1 ; t) & =\sum_{S T \in \mathcal{S} \mathcal{T}^{\lambda+\delta}(n, \bar{n})} t^{\operatorname{var}(S T)+\operatorname{bar}(S T)}(1+t)^{\operatorname{st}(S T)-n}, \\
& =\sum_{U A \in \mathcal{U} \mathcal{A}^{\lambda+\delta}(2 n)} t^{\operatorname{ssi}(U A)+\operatorname{bar}(U A)}(1+t)^{\operatorname{neg}(U A)}, \\
& =\sum_{C M \in \mathcal{C} \mathcal{M}^{\lambda+\delta}(2 n)} t^{\mathrm{ne}_{o}(C M)+\operatorname{se}_{e}(C M)+\operatorname{wgt}_{e}(C M)}(1+t)^{\mathrm{ns}(C M) .}
\end{aligned}
$$

Specialising further to the case $t=1$ gives

Corollary 6.6 Let $\lambda$ be a partition into no more than $n$ parts, let $\delta=(n, n-1, \ldots, 1)$, and let $m=\lambda_{1}+n$. Then

$$
2^{n^{2}} s p_{\lambda}(1)=\sum_{S T \in \mathcal{S} \mathcal{T}^{\lambda+\delta}(n, \bar{n})} 2^{\mathrm{str}(S T)-n}=\sum_{U A \in \mathcal{U} \mathcal{A}^{\lambda+\delta}(2 n)} 2^{\operatorname{neg}(U A)}=\sum_{C M \in \mathcal{C} \mathcal{M}^{\lambda+\delta}(2 n)} 2^{\mathrm{ns}(C M)} .
$$

Here $s p_{\lambda}(1)=s p_{\lambda}(1 ; 1)$ is the dimension of the irreducible representation of $s p(2 n)$ specified by $\lambda$, and it is known $[3,20,21]$ that this is given by

$$
s p_{\lambda}(1)=\prod_{1 \leq i<j \leq n} \frac{\lambda_{i}-i-\lambda_{j}+j}{j-i} \prod_{1 \leq i \leq j \leq m} \frac{\lambda_{i}+\lambda_{j}+n-i-j+2}{n+2-i-j} .
$$

However, as far as we know, no comparable product formula for $s p_{\lambda}(1 ; t)$ has yet been found.

Setting $\lambda=0$, so that $\mu=\delta=(n, n-1, \ldots, 1)$ in Theorems 6.2-6.4 gives

Theorem 6.7 Let $\delta=(n, n-1, \ldots, 1)$ and $m=n$. Then

$$
\begin{aligned}
D_{s p(2 n)}(x ; t) & =\sum_{S T \in \mathcal{S} \mathcal{T}^{\delta}(n, \bar{n})} t^{\operatorname{var}(S T)+\operatorname{bar}(S T)}(1+t)^{\operatorname{st}(S T)-n} x^{\mathrm{wgt}(S T)} \\
& =\sum_{U A \in \mathcal{U} A^{\delta}(2 n)} t^{\operatorname{ssi}(U A)+\operatorname{bar}(U A)}(1+t)^{\operatorname{neg}(U A)} x^{\mathrm{wgt}(U A)} \\
& =\sum_{C M \in \mathcal{C} \mathcal{M}^{\delta}(2 n)} t^{\mathrm{ne}_{o}(C M)+\operatorname{se}_{e}(C M)+\operatorname{wgt}_{e}(C M)}(1+t)^{\mathrm{ns}(C M)} x^{\mathrm{wgt}(C M)},
\end{aligned}
$$

where the summations are taken over all sp $(2 n)$-standard shifted tableaux $S T$ of shape $\delta$, all $2 n \times n$ UASMs whose column sums are all 1 and all $2 n \times n U$-turn square ice configuration matrices CM whose top-most element in each column is either NW or NS. 
Finally, setting $x_{1}=x_{2}=\cdots=x_{n}=1$ in Corollaries 6.5 and 6.6 gives

Corollary 6.8 Let $\delta=(n, n-1, \ldots, 1)$, then

$$
\begin{aligned}
(1+t)^{n^{2}} & =\sum_{S T \in \mathcal{S} \mathcal{T}^{\delta}(n, \bar{n})} t^{\operatorname{var}(S T)+\operatorname{bar}(S T)}(1+t)^{\operatorname{str}(S T)-n}, \\
& =\sum_{U A \in \mathcal{U} \mathcal{A}^{\delta}(2 n)} t^{\mathrm{sii}(U A)+\operatorname{bar}(U A)}(1+t)^{\operatorname{neg}(U A)}, \\
& =\sum_{C M \in \mathcal{C} \mathcal{M}^{\delta}(2 n)} t^{\mathrm{ne}_{o}(C M)+\operatorname{se}_{e}(C M)+\operatorname{wgt}_{e}(C M)}(1+t)^{\mathrm{ns}(C M)} .
\end{aligned}
$$

and

Corollary 6.9 Let $\delta=(n, n-1, \ldots, 1)$, then

$$
2^{n^{2}}=\sum_{S T \in \mathcal{S} \mathcal{T}^{\delta}(n, \bar{n})} 2^{\operatorname{str}(S T)-n}=\sum_{U A \in \mathcal{U} \mathcal{A}^{\delta}(2 n)} 2^{\operatorname{neg}(U A)}=\sum_{C M \in \mathcal{C} \mathcal{M}^{\delta}(2 n)} 2^{\mathrm{ns}(C M)} .
$$

\section{References}

1. D.M. Bressoud, Proof and Confirmations, MAA, Wash., D.C., 1999.

2. T. Eisenkölbl, "2-enumerations of halved alternating sign matrices," Sém. Loth. de Combin. B46c (2001), $11 \mathrm{pp}$.

3. N. El Samra and R.C. King, "Dimensions of irreducible representations of the classical Lie groups," J. Phys. A 12 (1979), 2317-2328.

4. A.M. Hamel and R.C. King, "Symplectic shifted tableaux and deformations of Weyl's denominator formula for $s p(2 n)$, , J. Algebraic Combin. 16 (2002), 269-300.

5. R.C. King, "Weight multiplicities for the classical Lie groups," in Lecture Notes in Physics, Springer, New York, 1976, vol. 50, pp. 490-499.

6. R.C. King and N.G.I.El-Sharkaway, Standard Young tableaux and weight multiplicities of the classical Lie groups, J. Phys. A 16 (1983), 3153-3177.

7. V.E. Korepin, "Calculation of norms of Bethe wave functions," Comm. Math. Phys. 86 (1982), 391-418.

8. G. Kuperberg, “Another proof of the alternating sign matrix conjecture," Internat. Math. Res. Notices 1996 (1996), 139-150

9. G. Kuperberg, "Symmetry classes of alternating sign matrices under one roof," Ann. of Math. (2) 156 (2002), $835-866$.

10. A. Lascoux, "Square ice enumeration," Sém. Loth. de Combin. B42 (1999), 15 pp.

11. E.H. Lieb, "Exact solution of the problem of the entropy of two-dimensional ice," Phys. Rev. Lett. 18 (1967), 692-694.

12. W.H. Mills, D.P. Robbins, and H. Rumsey, "Proof of the Macdonald conjecture," Invent. Math. 66 (1982), 73-87.

13. S. Okada, "Alternating sign matrices and some deformations of Weyl's denominator formula," J. Algebraic Comb. 2 (1993), 155-176.

14. J. Propp, "The many faces of alternating sign matrices," Disc. Math. and Th. Comp. Sci. July 2001.

15. J. Propp, http://www.math.wisc.edu/ propp/half-asm.

16. D.P. Robbins, "Symmetry classes of alternating sign matrices," arXiv:math. CO/008045 v1 5 Aug 2000

17. S Sundaram, "Tableaux in the representation theory of the classical Lie groups," in Invariant Theory and Tableaux, D Stanton (Ed.), IMA Springer-Verlag, New York, Vol. 19, 1989, pp. 191-225. 
18. T. Tokuyama, "A generating function of strict Gelfand patterns and some formulas on characters of general linear groups," J. Math. Soc. Japan 40 (1998), 671-685.

19. O. Tsuchiya, "Determinant formula for the six-vertex model with reflecting end," J. Math. Phys. 39 (1998), 5946-5951.

20. H. Weyl, "Theorie der Darstellung kontinuierlicher halb-einfacher Gruppen duch lineare Transformationen: I," Math. Z. 23 (1925), 271-309.

21. H. Weyl, "Theorie der Darstellung kontinuierlicher halb-einfacher Gruppen duch lineare Transformationen: II," Math. Z. 24 (1926), 328-376.

22. D. Zeilberger, "A proof of the alternating sign matrix conjecture," Elect. J. Comb. 3 (1996), R13. 